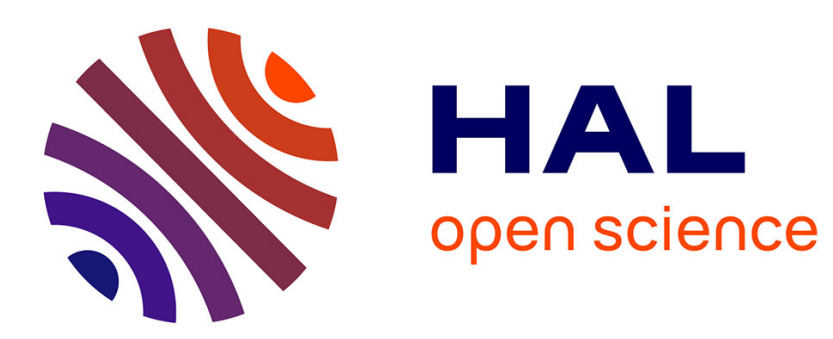

\title{
Coupling of sorption and deformation in soft nanoporous polymers: Molecular simulation and poromechanics
}

\author{
Mingyang Chen, Benoit Coasne, Dominique Derome, Jan Carmeliet
}

\section{To cite this version:}

Mingyang Chen, Benoit Coasne, Dominique Derome, Jan Carmeliet. Coupling of sorption and deformation in soft nanoporous polymers: Molecular simulation and poromechanics. Journal of the Mechanics and Physics of Solids, 2020, 137, pp.103830. 10.1016/j.jmps.2019.103830 . hal-02990005

\section{HAL Id: hal-02990005 https://hal.science/hal-02990005}

Submitted on 5 Nov 2020

HAL is a multi-disciplinary open access archive for the deposit and dissemination of scientific research documents, whether they are published or not. The documents may come from teaching and research institutions in France or abroad, or from public or private research centers.
L'archive ouverte pluridisciplinaire $\mathbf{H A L}$, est destinée au dépôt et à la diffusion de documents scientifiques de niveau recherche, publiés ou non, émanant des établissements d'enseignement et de recherche français ou étrangers, des laboratoires publics ou privés. 


\section{Coupling of Sorption and Deformation in Soft Nanoporous Polymers: Molecular Simulation and Poromechanics}

Mingyang Chen ${ }^{1, *}$, Benoit Coasne ${ }^{2}$, Dominique Derome ${ }^{3}$ and Jan Carmeliet ${ }^{1, *}$

1 Chair of Building Physics, Department of Mechanical and Process Engineering, ETH Zurich, 8093 Zurich, Switzerland

2 Univ. Grenoble Alpes, CNRS, LIPhy, 38000 Grenoble, France

3 Department of Civil and Building Engineering, Université de Sherbrooke, 2500, Sherbrooke, Canada

* Correspondence and requests should be addressed to M. C. (minchen@ethz.ch), J. C. (cajan@ethz.ch) 


\section{Highlights:}

1. Coupling mechanism of sorption and deformation is reviewed by molecular simulations.

2. Poromechanics model is developed based on the coupling mechanism at nanoscale.

3. Different types of sorption isotherms exhibited by different adsorbent-adsorbate systems are quantitatively explained by the developed model.

4. Influences of material properties on the coupled behavior of sorption and deformation are characterized. 


\begin{abstract}
The coupling of water sorption and deformation in soft nanoporous polymers is studied by means of statistical mechanics molecular simulation and the general framework of poromechanics. It is shown that the large amount of water adsorbed by soft nanoporous polymers under free swelling condition results from sorption-induced deformation, which generates more inter-chain space to accommodate water molecules. A poromechanical model is proposed to describe this coupled behavior from the molecular simulation data without any arbitrary fitting. More in detail, by taking into account the mechanical properties, sorption characteristics and structural information as a function of water loading, the model agrees with the molecular simulation and accurately captures the coupling mechanism. Using this model, it is also shown that distinct sorption and deformation behaviors can be observed depending on the material properties. On the one hand, small mechanical modulus, strong adsorbent-adsorbate interaction and significant coupling between sorption/deformation lead to Type II sorption isotherms (with larger sorption amount and sorption-induced deformation). On the other hand, Type I sorption isotherms with limited sorption amount and sorption-induced deformation are obtained for materials with opposite properties.
\end{abstract}

Keywords: nanoporous polymers, poromechanics, soft matter, molecular simulations 


\section{Introduction}

Sorption-induced deformation is a frequent occurrence in nanoporous polymers regardless of chemical components and micro-structures under study (Lee et al., 2019; Loh et al., 2005; Safari and van de Ven, 2016; Vandamme, 2019; Yang et al., 2018; Zhao et al., 2019). In nature, some plants use sorption-induced deformation for survival (Dawson et al., 1997). A plant material, wood, experiences significant swelling when exposed to moisture. In engineering, humidity-sensitive actuators, using nanoporous polymers as material, have been designed based on this feature (Yang et al., 2018). For polymer-based composites, performance can be affected when exposed to moisture as the swollen polymers after moisture uptake generally undergo deterioration in both stiffness and strength (Hahn, 1976; Loh et al., 2005). Deformations can in turn impact the sorption process, which is generally termed as 'mechanosorptive effect' (Chen et al., 2018; Kulasinski et al., 2015a). For instance, the sorption capacity can be greatly enhanced by the swelling of the polymers, which enables some polymer-based gels to harvest atmospheric water (Zhao et al., 2019). This sorption-induced deformation of polymers arises at the molecular level, where water molecules enter the inter-chain space, break the hydrogen bonds and deform the polymers. Upon deformation, the pores of the deformed polymers display a new energy landscape, which in turn impacts the sorption process (Kulasinski et al., 2015b, 2014b, 2014a). This two-way coupling effect has been observed not only in polymers but also in many other porous media, such as metal-organic frameworks (MOF) and aerogels (Gor et al., 2017; Günther et al., 2008; Neimark et al., 2010). However, as many nanoporous polymers undergo relative large sorption-induced deformation because of their low stiffness, the coupling effect is more noticeable in polymers (Anand, 2017; Derome et al., 2012; Lee et al., 2019; Weber et al., 2008; Zhao et al., 2019).

Interest in modeling the coupled behavior between sorption and deformation of nanoporous materials is growing and different models have been proposed. Some of them are built on thermodynamic approaches considering well-defined pore structures such as slit pore or cylinder pore (Balzer et al., 2017; Gor et al., 2018, 2017, 2013; Gor and Bernstein, 2016; 
Gor and Neimark, 2011). However, these approaches may not apply to polymers because they fail to capture the complex pore geometry in these materials. Indeed, pores in polymers refer to the morphologically distorted inter-chain space formed by disordered polymer chains. Another approach is to use the poromechanical framework, a macroscopic and continuum approach (Brochard et al., 2012; Kulasinski et al., 2015a; Vermorel and Pijaudier-Cabot, 2014; Zhang, 2018). The mechanical effects of sorption are integrated in poromechanics by considering an extra stress term, often referred to as sorption-induced stress. Some researchers relate the sorption-induced stress to a surface excess amount of the adsorbate and successfully model the sorption-induced deformation of materials such as coal (Zhang, 2018). For polymers, however, sorption cannot be described by surface covering, but rather by pore filling. Theories, such as Flory-Rehner theory, were built based on the concept of mixing polymer and liquid molecules rather than distinguishing the sorption surface (Flory and Rehner, 1943; Lai et al., 1991; Yu et al., 2018). The FloryRehner theory assumes that the elastic and mixing contributions to the free energy of swelling of dry polymer networks are separable and additive. The elastic contributions can be characterized knowing the mechanical constitutive relationship, while the mixing contribution is evaluated by considering the entropy and enthalpy of mixing. A solventpolymer interaction term is introduced in the theory, which is difficult to characterize directly by experiments or molecular simulations. Another way is to address the change in free energy of the system due to sorption by examining the sorption isotherm, as the state of the adsorbate can be fully characterized by the number of molecules adsorbed and its chemical potential. Following this line, Brochard et al. proposed a reformulation of poromechanics to account for sorption-induced stress in microporous solids as a function of the number of molecules adsorbed rather than by using the surface excess amount (Brochard et al., 2012). A similar strategy was adopted by Kulasinski et al. (Kulasinski et al., 2015a). Constitutive equations derived based on this strategy are valid for a disordered porous medium of nanometer size and can thus be applied to polymers.

Poromechanics, as a macroscopic approach, can be enriched by the information acquired from studies at the microscopic scale. As the coupling between deformation and sorption occurs at the molecular level, molecular simulations such as molecular dynamics (MD) and 
Grand Canonical Monte Carlo (GCMC) simulations are appropriate tools for its investigation (Chen et al., 2019, 2018; Kulasinski et al., 2014b, 2014a). Researches based on these numerical approaches can provide a clear microscopic picture of the coupling and capture the governing mechanisms. These numerical studies rely on molecular configurations and the simulations are computationally expensive and time-consuming. However, these microscopic mechanisms can be upscaled to the engineering scale using poromechanics, based on which a more general and efficient description of the coupled behavior can be expected.

Though the coupling between sorption and deformation is ubiquitous for polymers, it exhibits different features under different conditions. For instance, one can get different sorption isotherms under different mechanical conditions (Bakhshian and Sahimi, 2018; Chen et al., 2018). To better understand the interplay between sorption and mechanics in adsorbent-adsorbate systems with different sorption characteristics and mechanical properties, it is essential to develop a rational poromechanical model based on the microscopic coupling mechanism. In order to do this, in this paper, we first look at the coupling through molecular simulations, based on which a poromechanical model is proposed. The developed model allows us to study the sorption-induced deformation at different conditions and provides insights into the coupling phenomena. Finally, we demonstrate, using the developed model, the role of the different mechanical properties, sorption and porosity characteristics in the coupling and give corresponding physical interpretations

\section{Molecular Simulation}

\subsection{Model and Method}

In this study we use amorphous cellulose (AC) as the system representative of nanoporous polymers. To construct the molecular model of AC, cellobiose is firstly built as the repeat unit. The initial configuration of the cellulose chain is then generated using a Monte Carlo 
method within a periodic orthogonal box by employing the Amorphous Cell Module in Material Studio 8.0. The initial configuration of the AC has 4 chains and each chain has a degree of polymerization of 16. For the water molecules, we use SPC/E water model with the SHAKE algorithm (Ryckaert et al., 1977). The system is relaxed using MD simulation at constant temperature/stress with a Nose-Hoover thermostat/barostat with the help of LAMMPS (Large-Scale Atomic/Molecular Massively Parallel Simulator) (Plimpton, 1995). The temperature and stress are set to $300 \mathrm{~K}$ and $0 \mathrm{~Pa}$, respectively. The PCFF forcefield (Sun et al., 1994) is employed to describe both intramolecular and intermolecular interactions. The Ewald summation method is employed to account for long-range electrostatic interactions with periodic boundary conditions. The integration time step and thermostat relaxation time of MD are set to be $1 \mathrm{fs}$ and $500 \mathrm{fs}$ respectively. The relaxation is carried out for $2 \mathrm{~ns}$, after which the density converged well to a value of $1.40 \mathrm{~g} \mathrm{~cm}^{-3}$, comparable to the reported experimental value $1.48 \mathrm{~g} \mathrm{~cm}^{-3}$ (Chen et al., 2004). The prepared molecular configuration based on the approaches stated above is shown in dry and saturated states in FIG. 1 (a), with the box sizes of $2.73 \times 2.75 \times 3.07 \mathrm{~nm}^{3}$ and $3.08 \times 3.05 \times 3.35 \mathrm{~nm}^{3}$ for the dry and saturated state respectively. 
a

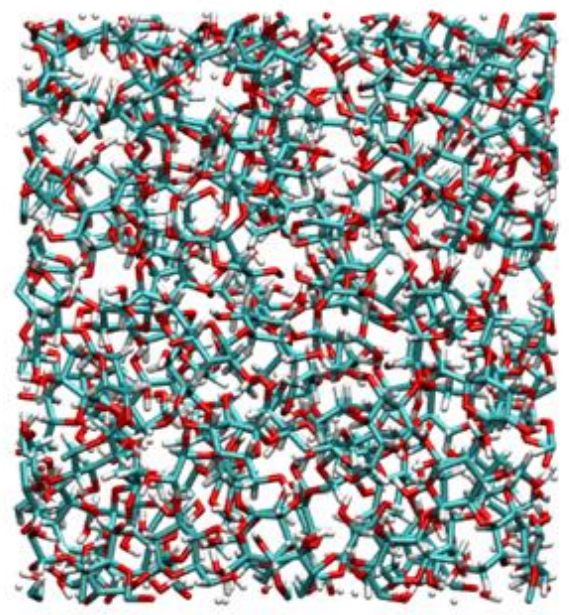

\section{b}

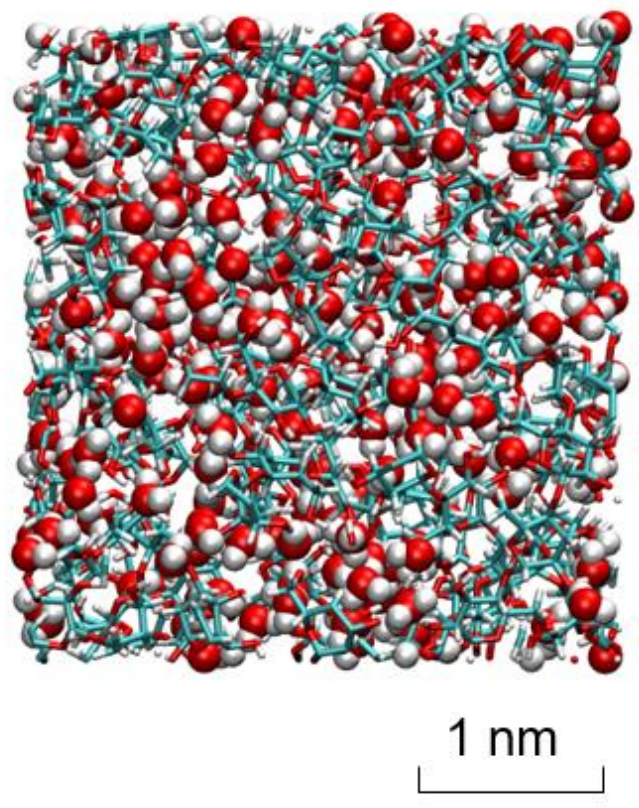

FIG. 1 Configurations in molecular simulations: (a) dry (unswollen) state at $\mathrm{RH}=0$; (b) saturated (swollen) state at $\mathrm{RH}=1$. Carbon, oxygen and hydrogen atoms are colored by cyan, red and white. Atoms of cellulose chains and water molecules are plotted in segments and spheres respectively. The temperature $T$ is $300 \mathrm{~K}$ and external stress $\sigma$ is 0 (stress free) for both cases.

To model the coupling between sorption and deformation, a hybrid scheme of molecular simulation that consists of GCMC and MD is employed to perform simulations in the socalled osmotic statistical ensemble $\mu \sigma T$ where $\mu$ is the chemical potential of water, $\sigma$ the stress applied to the cellulose material and $T$ the temperature. Compared to regular MD or GCMC, such a hybrid strategy enables one to model the sorption and deformation quasisimultaneously as it allows the system to deform during sorption. The hybrid molecular simulations are performed at $T=300 \mathrm{~K}$ using a Nose-Hoover thermostat/barostat and an anisotropic external stress $\sigma=0 \mathrm{~Pa}$. Each simulation block consists of 2,000 GCMC insertion/deletion attempts and $200 \mathrm{MD}$ timesteps. In total, $10^{5}$ blocks were first performed to equilibrate the system, followed by $2 \times 10^{4}$ additional blocks to accumulate statistics. One can refer to (Chen et al., 2019, 2018) for details and discussions on this hybrid technique. FIG. 1(b) shows the molecular configuration with swelling strain acquired from the simulation at saturated state. 


\subsection{Results}

FIG. 2 (a) compares the simulated water sorption isotherm with its experimental counterpart (Mihranyan et al., 2004). The sorption amount $n$ is defined as the number of water molecules per unit of initial (dry) volume, i.e. $n=N_{W} / V_{0}$, where $N_{W}$ is the number of adsorbed water molecule, $V_{0}$ is the initial volume of AC. The relative humidity RH is defined as $\mathrm{RH}=p / p_{0}$, where $p$ is the vapor pressure and $p_{0}$ is the saturation vapor pressure (1017 Pa for the SPC/E water model in our simulation (Chen et al., 2018)). The experimental data are shifted up by $n=+1.67 \mathrm{~nm}^{-3}$, an equivalent of 0.05 moisture content, to account for the presence of residual water in the 'dried' sample. One can refer to (Chen et al., 2018) for further information about the motivation for shifting up the experimental results. FIG. 2 (a) shows that the simulated sorption isotherm agrees well with experimental data. Both the simulation and experiment show that the sorption amount $n$ increases rapidly with RH in the low RH region and then less rapidly at medium RH. The data also show an inflection point after which significant subsequent sorption occurs, indicating that our molecular simulation grasps well the coupling between sorption and deformation. FIG. 2 (b) gives the sorption-induced strain of $\mathrm{AC}$ as a function of sorption amount. Here the strain is defined as volumetric strain, i.e. $\varepsilon=\left(V-V_{0}\right) / V_{0}$, where $V$ is the current sample volume. These data show that the strain is limited at very low sorption amount, after which the strain grows almost linearly with the sorption amount. These features indicate that the adsorbed water molecules at low RH mainly fill the initial porosity and thus do not lead to significant swelling. After the initial porosity is filled up, AC starts to swell and create more interchain space to accommodate water molecules. 

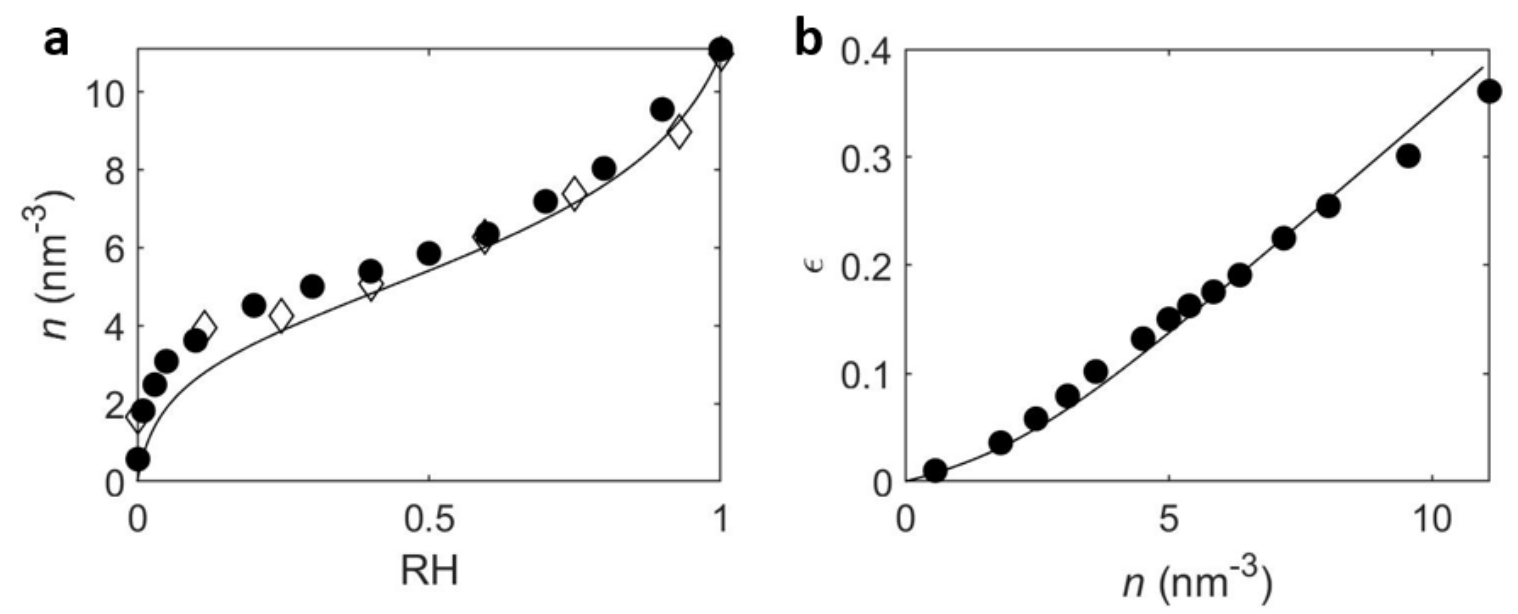

FIG. 2 (a) Sorption isotherms $n$ of water in AC at temperature $T=300 \mathrm{~K}$. (b) Relationship between strain $\varepsilon$ and sorption amount $n$. The closed circles and open diamonds correspond to molecular simulations and experiments (Mihranyan et al., 2004). The solid line represents the solutions from the poromechanics model.

Adsorption isotherms with such features are classified as Type II isotherm in the nomenclature of International Union of Pure and Applied Chemistry (IUPAC) (Thommes et al., 2015). This adsorption isotherm shape indicates unrestricted monolayer-multilayer adsorption (Sing, 1985). This explanation, however, does not apply to AC. According to (Chen et al., 2018), the pore size in AC is below $2 \mathrm{~nm}$, even for the swollen state, which makes it a microporous material (in fact, it can be shown that in such small cavities water is above its capillary critical temperature $T_{\mathrm{cc}}$ (Coasne et al., 2007; Schlaich and Coasne, 2019)). In such small pores, water molecules are strongly confined, and unrestricted multilayer adsorption cannot happen. Microporous materials such as activated carbons, molecular sieve zeolites, and certain porous oxides, display Type I isotherms, which are concave to the $\mathrm{RH}$ axis and approach a limiting value as $\mathrm{RH}$ approaches 1 . For such materials, the sorption amount grows fast at low RH because of the strong adsorbentadsorbate interaction. As the RH increases, the pore is getting filled, leading to a smaller increase in sorption amount upon further increasing RH. However, one distinct feature of AC compared to the other microporous materials mentioned above is its low mechanical modulus leading to a significant sorption-induced swelling. Swelling actually increases the inter-chain space (porosity) enhancing the sorption capacity. As a result, the sorption amount keeps increasing at high RH instead of being limited to a maximum value like in other microporous materials. 

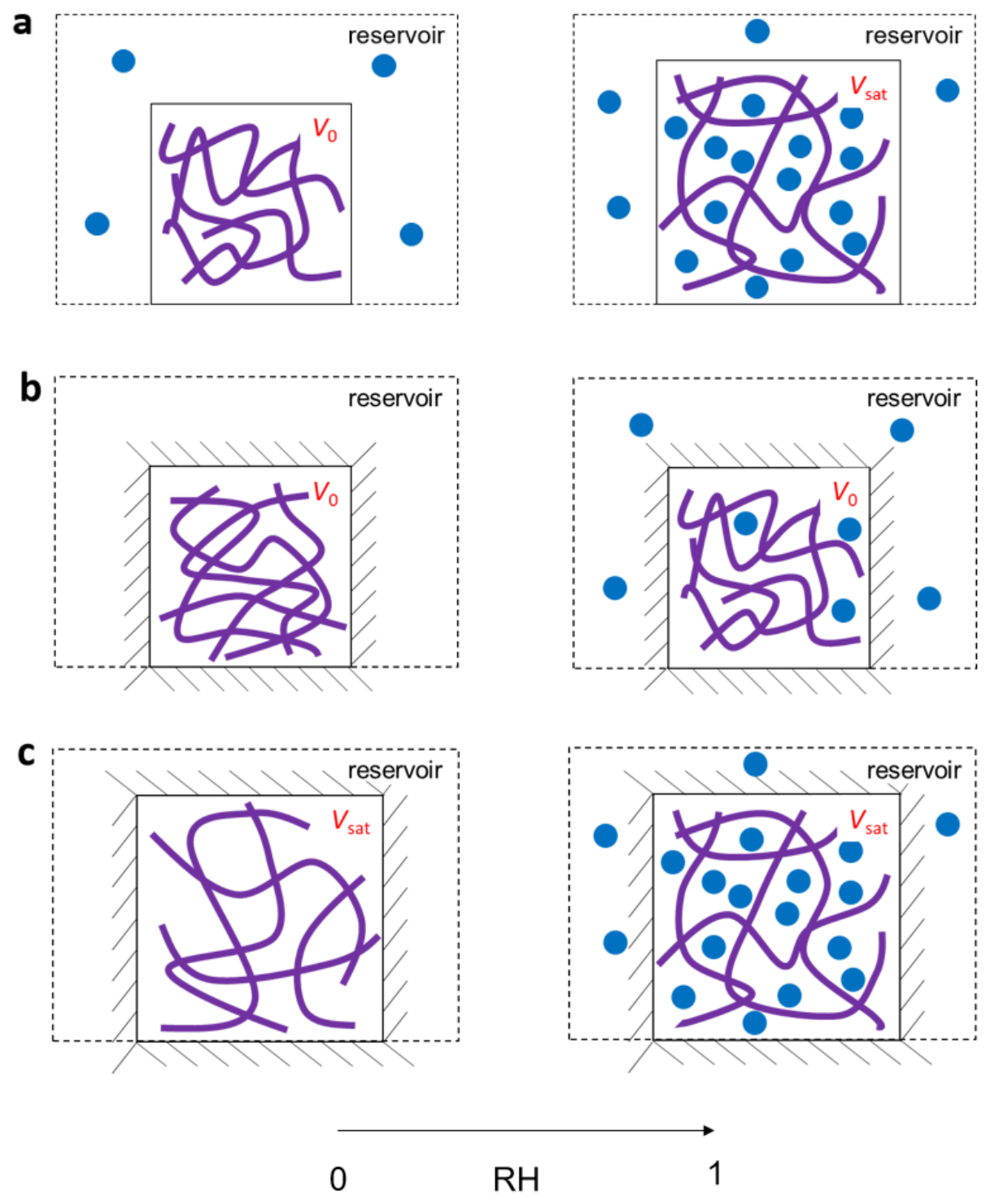

FIG. 3 Three boundary conditions considered when subjecting the polymer to a water vapor reservoir in molecular simulations and poromechanical model: (a) free swelling with system volume changing from $V_{0}$ at $\mathrm{RH}=0$ to $V_{\text {sat }}$ at $\mathrm{RH}=1$; (b) fixed displacement boundary conditions in unswollen state at volume $V_{0}$; (c) fixed displacement boundary conditions in swollen state at volume $V_{\text {sat. }}$ The blue dots and solid purple lines schematically represent water molecules and polymer chains respectively. The black solid line is the boundary of the system and the black dashed line represents the vapor reservoir. The black slash line represents the fixed boundary.

The explanation given above can be further verified by molecular simulations at conditions of constant volume. Instead of simulating at osmotic statistical ensemble $\mu \sigma T$ at free swelling case as shown in FIG. 3 (a), we conduct the simulation at the grand canonical 
ensemble $\mu V T$, where the volume is constant and no deformation is allowed. Two solid configurations are considered: the unswollen state with volume of $V_{0}$ and maximum swollen states with volume of $V_{\text {sat }}$ as schematized in FIG. 3 (b) and (c). The results are plotted in FIG. 4 (a); we get adsorption isotherms for both cases that follow the Type I adsorption isotherm. The difference between the two adsorption isotherms comes from the different initial porosities. In the swollen state, the larger inter-chain space leads to larger porosity, which can accommodate more water molecules and thus has a higher sorption capacity. The Type I isotherms obtained here are consistent with other stiff microporous materials with limited sorption-induced deformations (Fomkin, 2005; Jin et al., 2014; Yakovlev et al., 2004). In the absence of significant deformation, the sorption process of $\mathrm{AC}$ would have followed the behavior of regular microporous materials. The comparison between FIG. 2 (a) and FIG. 4 (a) clearly displays the pronounced influence of deformation on the sorption process of AC. In practice, the sorption isotherms are often used to derive pore size distribution based on the assumption of constant pore sizes upon sorption (Dollimore and Heal, 2007; Groen et al., 2003). Our results here indicate that one has to take the coupling between sorption and deformation into account for soft materials showing significant sorption-induced deformation.
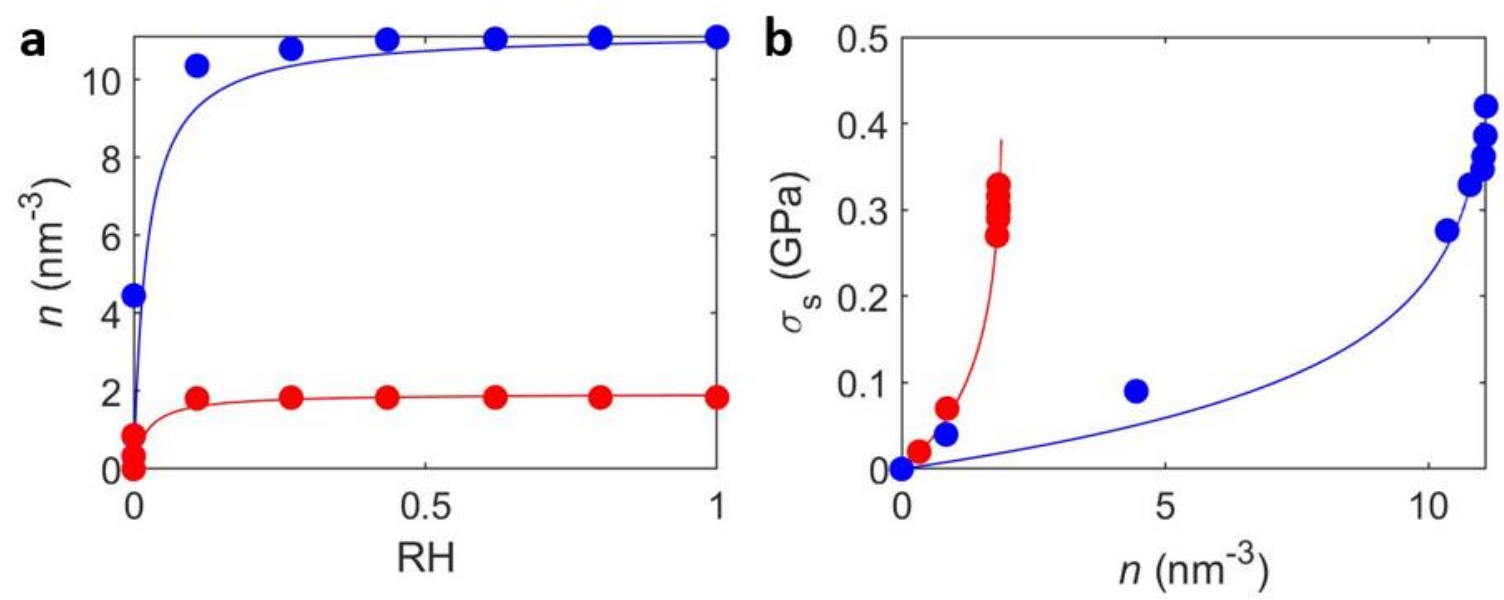

FIG. 4 (a) Sorption isotherms $n$ of water in AC at fixed unswollen (red) and swollen (blue) states with temperature $T=300 \mathrm{~K}$ (b) Relationship between sorption-induced stress $\sigma_{\mathrm{s}}$ and sorption amount $n$ at fixed unswollen (red) and swollen (blue) states. The closed circles and solid lines represent molecular simulations and poromechanics solutions respectively. 
In these simulations with constrained deformation, the variation of sorption amount leads to change of stress applied to the solid. As shown in FIG. 4 (b), we measure the stress change (volumetric) as a function of the sorption amount. This stress is termed as sorptioninduced stress in some literature (Gor et al., 2018). Considering the fact that the maximum vapor pressure $p_{0}$ in our simulation is only $1017 \mathrm{~Pa}$, the sorption-induced stress is much larger than the vapor pressure in the reservoir. One can refer to (Long et al., 2012) for a more detailed discussion on this pressure enhancement induced by nano-confinement. For both cases, the sorption-induced stress is convex to the sorption amount axis. This indicates that the adsorbed water molecules at low RH, which mainly fill the initial porosity, do not lead to a built up of significant stresses. After the free volume is exhausted, water molecules confined in the pores lead to severe crowding so that steric repulsion prevents further adsorption. As a result, further increase of the number of water molecules leads to a larger increase in sorption-induced stress. FIG. 4 (b) also shows that the swollen AC adsorbs more water molecules to achieve a similar sorption-induced stress as in unswollen AC. The swollen AC with larger porosity needs to host more water molecules to achieve the same degree of crowding.

Though molecular simulation results above agree with the experiments and show the coupling mechanism at molecular level, they are subjected to certain limitations. First, the results obtained here are specific to AC. As a matter of fact, not all polymers exhibit Type II sorption isotherms upon free swelling condition. Type I sorption isotherms are also reported (Meng and Weber, 2014; Park et al., 2007). A model that is able to handle different conditions is needed. Second, the molecular simulation results provide a qualitative explanation of the coupling mechanism but a quantitative analysis based on energy balance is still needed. Last but not least, molecular simulations are generally unable to characterize the individual (i.e. independent, without coupling) contributions of different physical properties such as modulus and sorption capacity. We thus need to turn to poromechanics to overcome these limitations and gain better knowledge of the coupled behaviors.

\section{Poromechanics}




\subsection{Derivation}

Consider a porous material in contact with a vapor reservoir. The fluid phase has to be treated in the grand canonical ensemble as it can exchange molecules with the reservoir. For the fluid at equilibrium, the state can be fully determined by its temperature $T$, volume $V_{f}$ and chemical potential $\mu_{k}$ of the $k$-th component. An infinitely small change in the grand potential $\Omega_{f}$ writes:

$$
\mathrm{d} \Omega_{f}=-S_{f} \mathrm{~d} T-P \mathrm{~d} V_{f}-\sum_{k} N_{k} \mathrm{~d} \mu_{k}
$$

where $S_{f}$ and $P$ are the fluid entropy and pressure, $N_{k}$ is the number of molecules of the $k$ th component. In contrast, the solid phase, having a volume of $V_{0}$, does not exchange molecules with the environment and must therefore be treated in the canonical ensemble, where the Helmholtz free energy $F_{\mathrm{s}}$ is minimized at equilibrium. An infinitely small change in the Helmholtz free energy balance is given by:

$$
\mathrm{d} F_{s}=-S_{s} \mathrm{~d} T+\boldsymbol{\sigma} V_{0} \mathrm{~d} \boldsymbol{\varepsilon}+P \mathrm{~d} V_{f}
$$

where $S_{s}$ is the solid entropy while $\boldsymbol{\sigma}$ and $\boldsymbol{\varepsilon}$ are the stress and strain tensors respectively. The term $P \mathrm{~d} V_{f}$ involves the work done by the fluid on the solid. The total grand potential of the solid-fluid system $\Omega$ can be considered as the summation of the grand free energy of the fluid and the free energy of the solid, i.e. $\Omega=\Omega_{f}+F_{s}$. The grand potential density $\omega$, which is defined as $\omega=\Omega / V_{0}$, can be written as:

$$
\mathrm{d} \omega=-s \mathrm{~d} T+\boldsymbol{\sigma} \mathrm{d} \boldsymbol{\varepsilon}-\sum_{k} n_{k} \mathrm{~d} \mu_{k}
$$

where $s$ is the entropy density of the system, $n_{k}$ the number of fluid molecules per volume of the $k$-th component. The use of Maxwell relation in Eq. (3) gives: 


$$
\left.\frac{\partial \boldsymbol{\sigma}}{\partial \mu_{k}}\right|_{\varepsilon, T, \mu_{k^{\prime} \neq k}}=\left.\frac{\partial n_{k}}{\partial \boldsymbol{\varepsilon}}\right|_{T, \mu_{k^{\prime}}}
$$

Integration with respect to the chemical potential gives:

$$
\boldsymbol{\sigma}=\boldsymbol{\sigma}_{0}-\left.\frac{\partial}{\partial \boldsymbol{\varepsilon}}\left[\int_{(-\infty, \ldots,-\infty)}^{\mu_{1}, \ldots, \mu_{\kappa}} \sum_{k} n_{k} \mathrm{~d} \mu_{k}\right]\right|_{T, \mu_{k}}
$$

where $\boldsymbol{\sigma}_{0}$ is the stress that prevails in absence of fluid. Here we consider the sorptioninduced deformation as isotropic. Coupling between sorption and deformation is also considered isotropic and the stress deviator is assumed to be unaffected. Under isothermal conditions, by considering volumetric stress and strain and a single fluid, Eq. (5) is reduced to:

$$
\sigma=\sigma_{0}(\varepsilon)-\frac{\partial}{\partial \varepsilon} \int_{-\infty}^{\mu} n \mathrm{~d} \mu
$$

where $\sigma$ and $\varepsilon$ are the volumetric stress and strain respectively. In the following part, the stress and strain deviators are not considered. Without further notification, the terms stress and strain refer to volumetric stress and strain respectively from now on.

To apply this thermodynamics-based derivation to a specific material, the sorption isotherm and the mechanical constitutive relationships have to be specified. First we consider the sorption isotherm, which is a function of both chemical potential and strain, i.e., $n=n(\mu, \varepsilon)$. Strain can influence the sorption process in many ways. Straining the material leads to a change in pore volume, as shown in the molecular simulations of AC; thus, the strained material will show a different sorption capacity compared to the undeformed material. Also, strain can change the density of adsorption sites and modify the energy landscape within the pore, leading to a change in sorption amount. Considering the fact that the deformation is large in $\mathrm{AC}$ and that sorption is greatly enhanced by the 
enlarged pore capacity, we assume that the influence of strain on the sorption isotherm solely comes on the enlarged sorption capacity. Then the sorption amount can be written as

$$
n(\varepsilon, \mu)=\frac{\phi(\varepsilon, \mu) n_{0}(\mu)}{\phi_{0}}
$$

where $n_{0}(\mu)$ is the 'baseline' sorption isotherm, which corresponds to the sorption isotherm at zero strain. $\phi(\varepsilon, \mu)$ is the current Lagrangian porosity depending on strain and chemical potential. $\phi_{0}$ is the initial (undeformed) porosity corresponding to the dry state of the solid. The dependence of the current porosity on strain can be written based on the first-order expansion as:

$$
\phi(\varepsilon, \mu) \approx[1+C(\mu) \varepsilon] \phi_{0}
$$

where $C(\mu)$ is a parameter characterizing the dependence of porosity on strain. As shown in (Coasne et al., 2014), the exact dependence for $\phi(\varepsilon, \mu)$ can be considered and the poromechanics model as described in Eq. (6) can be solved in an iterative fashion. According to (Brochard et al., 2012), the dependence of $C(\mu)$ on chemical potential is not significant for amorphous materials. This also applies to nanoporous polymers as the polymer chains are in the amorphous state. Following this line, we neglect the dependence of $C(\mu)$ on chemical potential and simplify it into a constant $C$. Eqs. (7) and (8) leads to

$$
n(\varepsilon, \mu)=[1+C \varepsilon] n_{0}(\mu)
$$

In Eq. (9), $C$ links sorption and strain and is termed the sorption-strain coupling constant in some literature (Zhang, 2018). For different adsorbent-adsorbate systems, $C$ can be different depending on the coupling between sorption and deformation. To determine $C$ for $\mathrm{AC}$, we consider the fact that polymeric chains are not cross-linked so that the bulk material shows a much lower stiffness compared to the stiffness of cellulose chains. As a result, the 
cellulose chains will be barely strained and the deformation mainly comes from the change of the inter-chain space or change in porosity. Assuming the deformation of the solid is negligible, strain of the system can be expressed as:

$$
\varepsilon \approx \phi(\varepsilon)-\phi_{0}
$$

Combining Eqs. (7), (9) and (10) gives $C=1 / \phi_{0}$. These simplifications on $C$ can be verified by comparing with the molecular simulations. The porosity of the molecular model is probed using the method given in (Kulasinski et al., 2015b). As shown in FIG. 5 (a), the porosity-strain relationship with $C=1 / \phi_{0}$ agrees with that measured in the molecular model. This indicates that $C=1 / \phi_{0}$ is a reasonable simplification when considering the change of porosity with strain for AC. One should note that relating $C$ to the porosity is not compulsory. The simplification $C=1 / \phi_{0}$ only applies when all the deformation of the system is caused by the change of pore (inter-chain) space. For a more general consideration, $C$ can be directly determined based on the molecular simulation results with the help of Eq. (9).
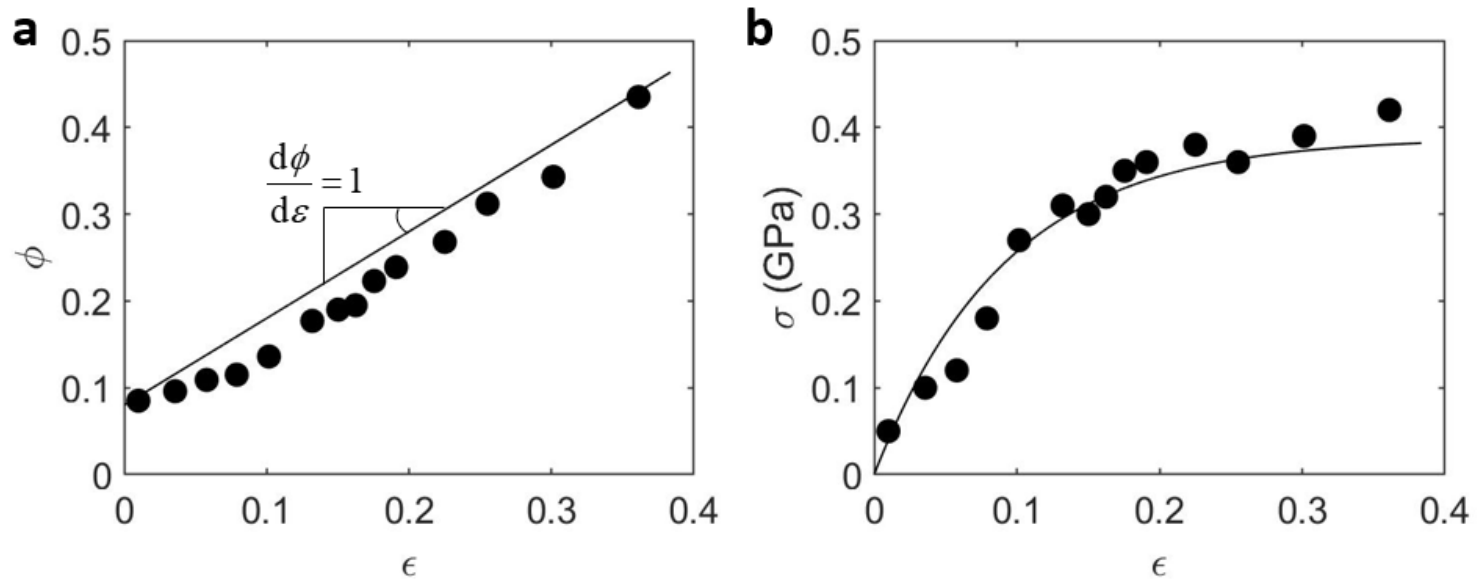

FIG. 5 (a) Dependence of porosity $\phi$ on strain $\varepsilon$ based on molecular simulations (closed circle) and Eq. (10) 
(solid line). (b) Relationship between stress $\sigma_{0}$ and strain $\varepsilon$ of the solid part. The closed circles are data extracted form molecular simulations, which is fitted by Eq. (15) plotted as the solid line.

The other term to be determined is the 'baseline' sorption isotherm $n_{0}(\mu)$ of the undeformed solid. As shown in the last section, the molecular simulation predicts a Type I sorption isotherm in the unswollen case that can be described using the Langmuir adsorption model:

$$
n_{0}=\frac{n_{0}^{0} B p}{1+B p}
$$

where $n_{0}{ }^{0}$ and $B$ are sorption parameters. $n_{0}{ }^{0}$ defines the sorption capacity while $B$ defines the slope of the adsorption isotherm at very low vapor pressures $\left(p / p_{0}=\mathrm{RH}\right)$. The two parameters are calibrated by fitting the sorption isotherm of the unswollen model. The determined $n_{0}{ }^{0}$ and $B$ values are given in Table 1. Combining Eq.(6), Eq. (9) and (11), and taking into account $\mathrm{d} \mu=(R T / p) \mathrm{d} p$, we have:

$$
\sigma+\sigma_{\mathrm{s}}=\sigma_{0}(\varepsilon)
$$

where $\sigma_{\mathrm{s}}$ is the sorption-induced stress expressed as:

$$
\sigma_{\mathrm{s}}=C R T n_{0}^{0} \ln (1+B p)
$$

Apart from the sorption isotherm, we should also specify the mechanical constitutive relationship, i.e. how stress depends on strain. As shown in (Kulasinski et al., 2015b), a positive (swelling) strain can increase inter-chain distance of the $\mathrm{AC}$, resulting in a decrease in the tangential bulk modulus. The tangential bulk modulus is thus assumed to decrease with increasing porosity, which can be written as:

$$
\mathrm{d} \sigma_{0}=K_{0} \exp (-b \phi) \mathrm{d} \varepsilon
$$


where $K_{0}$ is the bulk modulus at zero porosity, whereas $b$ is a parameter controlling the decaying behavior of the tangential modulus versus porosity. Specially, the material is linear elastic if $b=0$. Combining Eqs. (10) and (14) and integrating over $\varepsilon$, we have:

$$
\sigma_{0}=K_{0}[1-\exp (-b \varepsilon)] \exp \left(-b \phi_{0}\right) / b
$$

Finally, substituting Eqs. (13) and (15) into Eq. (12), the sorption-induced strain can be determined. Upon this point, a poromechanical framework covering both sorption-induced deformation and mechanosorptive effect has been established to describe the two-way coupling between sorption and deformation.

Table 1 Parameters used for the poromechanics model

\begin{tabular}{ccccccc}
\hline $\begin{array}{c}\text { parameter } \\
\text { units }\end{array}$ & $\begin{array}{c}n_{0}^{0} \\
\left(\mathrm{~nm}^{-3}\right)\end{array}$ & $\begin{array}{c}B \\
\left(\mathrm{~Pa}^{-1}\right)\end{array}$ & $\begin{array}{c}K_{0} \\
(\mathrm{GPa})\end{array}$ & $\begin{array}{c}\phi_{0} \\
-\end{array}$ & $\begin{array}{c}C \\
-\end{array}$ \\
\hline value & 1.94 & 0.0433 & 9.98 & 10.8347 & 0.081 & 12.36 \\
\hline
\end{tabular}

To calibrate $K_{0}$ and $b$, we extract the stress-strain curve in the solid part from molecular simulations with the following method. At each sorption amount (with different swelling strains) of the free swelling case, the system is fixed and all water molecules are deleted, then the stress prevailing in the solid is measured. Combining the stress-strain pairs for all the different sorption amounts, we get the stress-strain relationship of the solid part as seen in FIG. 5 (b). $K_{0}$ and $b$ are determined by fitting Eq. (15) against the data with the least square method; their values are given in Table 1.

\subsection{Results}

FIG. 2 (a) gives the sorption isotherm under free swelling stemming from the poromechanical model. The predicted data show good agreement with both molecular simulations and experiments. The steeper sorption changes at low and high RHs framing 
the flatter section at medium RH are well captured by the poromechanical model. FIG. 2 (b) shows the calculated strain curve in comparison with the molecular simulation results. Both results show a smaller slope at very low sorption amount and a larger slope for the remaining sorption amounts. These comparisons show that, despite the assumptions made when conducting the derivation, the poromechanical model captures the governing mechanism of the coupled behavior between sorption and deformation.

The poromechanical model is versatile as it is easy to use for the study of the coupled behavior under different conditions. We further compare the poromechanical results with molecular simulations for the fixed unswollen and swollen states respectively. The sorption isotherms are plotted in FIG. 4 (a). The Langmuir isotherm is seen to adequately describe the sorption isotherm at fixed strain. According to Eq. (7), the sorption amount is proportional to the current porosity. Thus if we scale the sorption amount with the porosity, the data from unswollen, swollen and free swelling cases should all collapse. That is what we see in FIG. 6 (a), therefore showing that the sorption isotherm for different cases can be described by a single Langmuir isotherm given by Eq. (11). The data in FIG. 6 (a) suggest that the poromechanical solution agrees best with the unswollen case. This is because the parameters in the Langmuir adsorption isotherm are calibrated using data from the unswollen case in this work. 

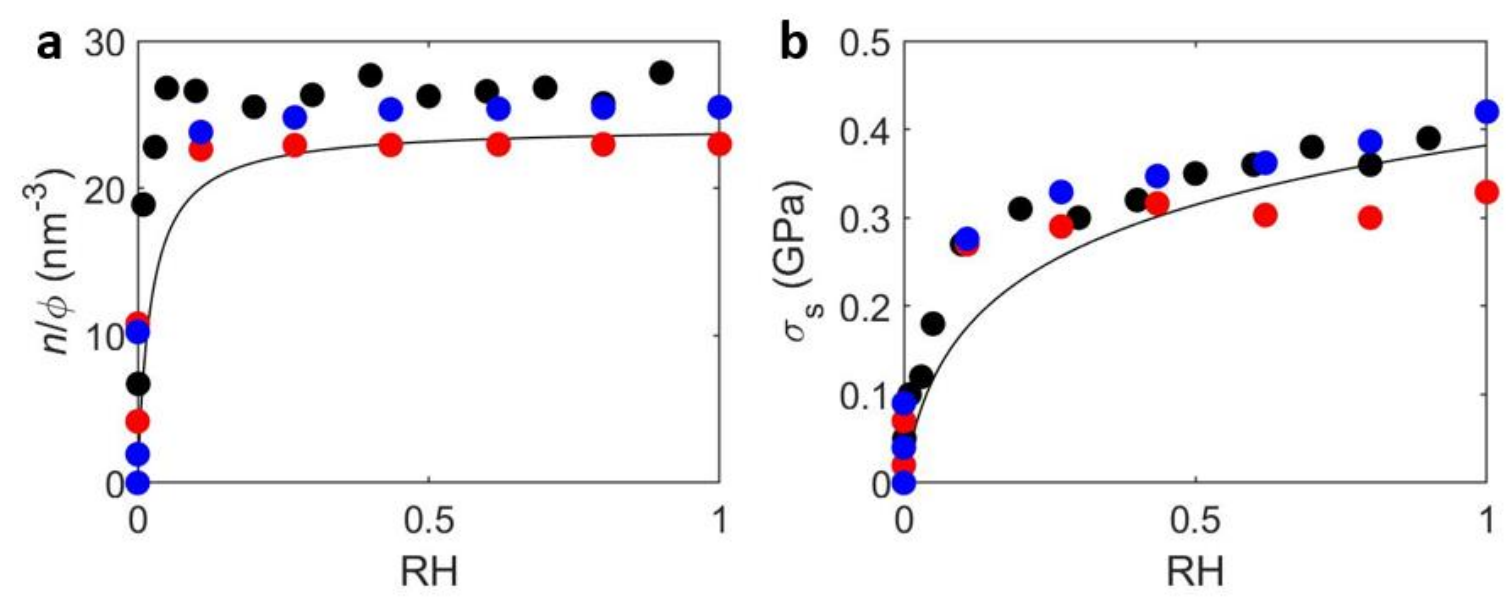

FIG. 6 (a) Sorption isotherms $n$ scaled by porosity $\phi$, i.e. $n / \phi$. (b) Dependence of sorption-induced stress $\sigma_{\mathrm{s}}$ on RH. The black, red and blue closed circles represent the free swelling, unswollen, and swollen cases respectively. The solid line represents the poromechanics solutions

The sorption-induced stress can be easily calculated from Eq. (13). Also, the sorptioninduced stress can be extracted from molecular simulations. In this case, the stress tensor in molecular simulation is calculated based on the viral expression. For both the unswollen and swollen cases, the sorption-induced stress $\sigma_{\mathrm{s}}$ is taken as the stress change of the system upon sorption. However, this strategy cannot be applied to the free-swelling case as the stress of the system is always 0 due to the free deformation. To extract the sorption-induced stress $\sigma_{\mathrm{s}}$, we delete water molecules and measure the stress left in solid skeleton, which is taken as $\sigma_{\mathrm{s}}$ for the free swelling case. FIG. 4 (b) shows that the poromechanical solutions follow the same trend as the molecular simulation results for both the unswollen and swollen cases. It is worth noting that, according to Eq. (13), the sorption-induced stress is strain independent, which means that the stress obtained for different strains should collapse when plotted against RH. As shown in FIG. 6 (b), though the stresses calculated by molecular simulations do not strictly follow coincide, they are scattered around the poromechanical solutions. Deviations may arise from assumptions made on the coupling constant $C$. The strain-independent feature of sorption-induced stress comes from the fact that we simplify $C$ to be a constant independent of strain. In reality, $C$ is a function of both chemical potential and strain and the sorption-induced stress will show some strain dependence in molecular simulations. However, considering the fact that the difference between the sorption-induced stress with the minimum (unswollen case) and the maximum 
(swollen case) strain is at maximum 25\%, we conclude that independence of $C$ on strain is a reasonable assumption (again, see (Coasne et al., 2014) for a treatment where such approximations are avoided).
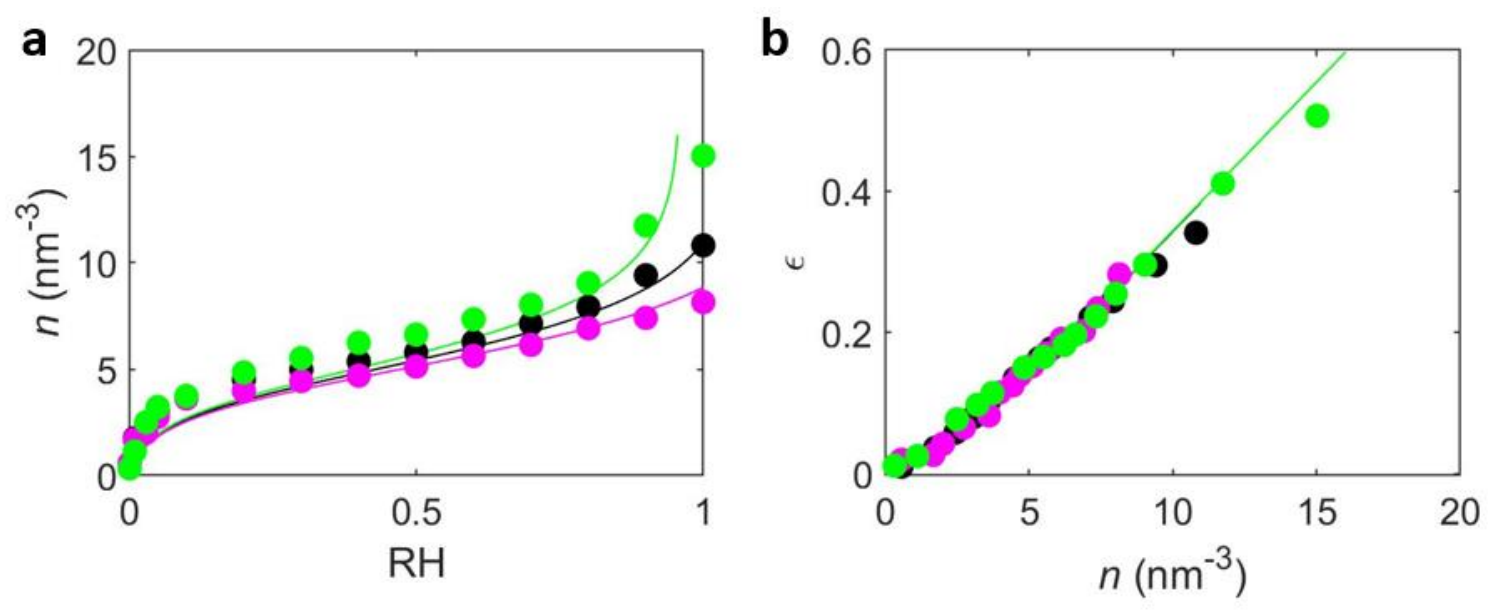

FIG. 7 Comparison of (a) sorption isotherm and (b) strain-sorption amount relationship between poromechanical solutions (solid line) and molecular simulations (closed circle) at three different loading conditions: $\sigma=-10 \mathrm{MPa}$ (magenta), 0 (black) and 10MPa (green).

To further verify the validity of the poromechanical model, we compare results with the molecular simulations at different loading conditions. Two additional molecular simulations at $\mu \sigma T$ ensemble are conducted for stresses $\sigma=10 \mathrm{MPa}$ and $\sigma=-10 \mathrm{MPa}$ (corresponding to the sorption process under tension and compression respectively). For the poromechanical solution, the effect of external loading can be easily taken into account by setting $\sigma$ to the corresponding values in Eq. (13). Comparisons between poromechanical solutions and molecular simulations at three different loading conditions $(\sigma=-10 \mathrm{MPa}, 0$, 10MPa) are shown in FIG. 7. In FIG. 7 (a), the sorption amount increases as the loading turns from compression to tension. The tension loading increases the pore space so that sorption is promoted (more free volume available). This feature is successfully captured by the poromechanical model, which shows good agreement with the molecular simulations in all three loading conditions. Moreover, FIG. 7 (b) shows that the data from the three different loading conditions collapse when plotting the strain versus sorption amount for both molecular simulations and poromechanical solutions. This is because the slope in strain-sorption amount curve is mainly determined by the dependence of the 
current porosity on the strain, i.e. $C$ in Eq. (8). The influence of mechanical loading on $C$ for $\mathrm{AC}$ is not significant.

One feature of the poromechanical model is that the sorption isotherm cannot reach $\mathrm{RH}=1$ at high tensile loading. This feature can be seen in FIG. 7 (a) for the case $\sigma=10 \mathrm{MPa}$ : the sorption amount diverges before $\mathrm{RH}$ reaches 1 . This corresponds to the fact that chains will just unfold and lose their stiffness and interactions under high tensile loading and high moisture content. This feature comes from the mechanical constitutive relationship employed in this work, i.e. Eq. (15). According to Eq. (15), there exists a maximum stress that the solid can sustain which is $\sigma_{\max }=K_{0} \exp \left(-b \phi_{0}\right) / b$. If the total stress combining the sorption-induced stress and external stress is larger than $\sigma_{\max }$, the solution yields an infinite strain, equivalent to an evaporation of the material. It is worth noting that this feature is not just specific to the tension loading. As a matter of fact, even at zero mechanical loading, i.e. $\sigma=0$, this feature can emerge by adjusting model parameters listed in Table 1 to make the solid very soft or the sorption very strong. This point will be mentioned in the Discussion section below. In reality, this phenomenon is observed in some materials (Barreiro et al., 2010) but it does not apply to all nanoporous polymers. In molecular simulations, RH=1 can be reached at this mechanical loading. This deviation can be attributed to two major reasons. First, polymers stop weakening at large strains because of the entanglement and straightening of polymer chains (Bharadwaj et al., 2017; Jaspers et al., 2014). In other words, the mechanism governing the mechanical constitutive relationship changes at large strain. However, the constitutive relationship in Eq. (15) is proposed and calibrated based on the stress-strain curve of molecular simulations with strain below 0.4 (as shown in FIG. 5 (b)) - the weakening because of the enlarged interchain space is still the governing mechanism. Thus, features of AC at large strain cannot be captured by the Eq. (15). Second, pore size can become very large at large strains, leading to a change of sorption behavior. Thus, the Langmuir isotherm may become inappropriate in describing the sorption behavior for large pores with large strains. Thus, if one wants to address the coupling phenomenon at high tensile loading under the framework of poromechanics, a more comprehensive mechanical constitutive relationship capable of addressing the stiffening effect at high strain needs to be included (Xiang et al., 
2019). Moreover, the sorption isotherm needs to be recalibrated considering the possible change of sorption physics within large pores due to the large strain. However, although the current poromechanical model cannot recover the sorption isotherm over the entire $\mathrm{RH}$ range for high tension loading, it successfully captures the strong convexity at high RH and agrees with the molecular simulations at low and medium RH.

\section{Discussion}

\subsection{Influence of Material Properties}

By comparing with molecular simulations, we find that the poromechanical solutions capture the governing mechanism of the coupled behavior of sorption and deformation. We proceed in what follows to a parametric analysis studying how the material properties influence the coupled behavior. There are three groups of material-related parameters in the poromechanical model: the mechanical properties $K_{0}$ and $b$, the sorption characteristics $n_{0}{ }^{0}$ and $B$ and the structural information $\phi_{0}$ and $C$. In this section we discuss in detail the influence of these material parameters on the coupled behavior with the help of the poromechanical model.

First, we look at the mechanical properties. In the poromechanical model, the mechanical behavior of the solid is governed by two parameters: the modulus at zero porosity $K_{0}$ and the weakening parameter $b$. A solid with lower $K_{0}$ and larger $b$ means a softer material. Sorption isotherms at different $K_{0}$ values are plotted in FIG. 8 (a). All the other parameters are kept constant. As $K_{0}$ increases, the sorption amount is lower for the same RH. Indeed, a larger $K_{0}$ corresponds to a stiffer material with less sorption-induced deformation. As a result, there is less additional pore space created by swelling to accommodate water molecules which finally leads to smaller sorption amount. The sorption isotherms change from Type II to Type I as $K_{0}$ increases. For a better description of the transition, we define Type I and Type II as follows: a sorption isotherm is Type I if the sorption isotherm is 
concave for all the RH (vapor pressure) from 0 to 1 and Type II if the sorption isotherm turns from concave to convex below $\mathrm{RH}=1$ :

$$
\begin{array}{ll}
\text { Type-I: } & \frac{\mathrm{d}^{2} n}{\mathrm{~d} p^{2}}<0 \quad \forall p \in\left[0, p_{0}\right] \\
\text { Type-II : } & \left.\frac{\mathrm{d}^{2} n}{\mathrm{~d} p^{2}}\right|_{p=p_{C}}=0,\left.\frac{\mathrm{d}^{2} n}{\mathrm{~d} p^{2}}\right|_{p<p_{C}}<0,\left.\frac{\mathrm{d}^{2} n}{\mathrm{~d} p^{2}}\right|_{p>p_{C}}>0 \exists p_{C} \in\left[0, p_{0}\right]
\end{array}
$$

By combing the classification for Type-I and Type-II in Eq. (16), we find that there is one critical state characterizing the transition between the two types of isotherms when $p_{\mathrm{C}}=p_{0}$, i.e., $\mathrm{d}^{2} n / \mathrm{d} p^{2}=0$ at $p=p_{0}$. This means that the sorption isotherm turns from concave to convex just at $\mathrm{RH}=1$. Correspondingly we can define a critical modulus $K_{\mathrm{c}}$ at this critical state. The physical meaning of $K_{\mathrm{c}}$ is that a Type I sorption isotherm results from materials with a modulus above $K_{\mathrm{c}}$ while a Type II isotherm for materials with a modulus below $K_{\mathrm{c}}$. In other words, one can use this critical value to characterize what type of sorption isotherms is to be expected for a given adsorbent-adsorbate system.

As mentioned in the Results section, one feature of the poromechanical model is that it gives infinite sorption amounts before $\mathrm{RH}=1$ at certain mechanical conditions. Here for $K_{0}$ specifically, a very low $K_{0}$ can lead to this condition. This means that, for very soft materials, the solid cannot sustain the sorption-induced stress yielding very large strains. Thus, we can define another critical value of $K_{0}, K_{\mathrm{cc}}$, below which the strain goes to infinity when approaching $\mathrm{RH}=1$. The poromechanical model is valid through the entire $\mathrm{RH}$ range, i.e., from 0 to 1 , only if $K_{0}>K_{\mathrm{cc}}$. With the two critical values $K_{\mathrm{c}}$ and $K_{\mathrm{cc}}$, we define three regions in FIG. 8 (a) corresponding to three distinct sorption isotherms indicated in FIG. 8 (g). Region I (grey) with $K_{0}>K_{\mathrm{c}}$ and Region II (pink) with $K_{\mathrm{c}}>K_{0}>K_{\mathrm{cc}}$ correspond to Type I and Type II sorption isotherms respectively. Region III (white), with $K_{0}<K_{\mathrm{cc}}$ corresponds to poromechanical solutions with infinite strains below $\mathrm{RH}=1$. The lower bound of Region I corresponds to the sorption isotherm of the undeformed case. 

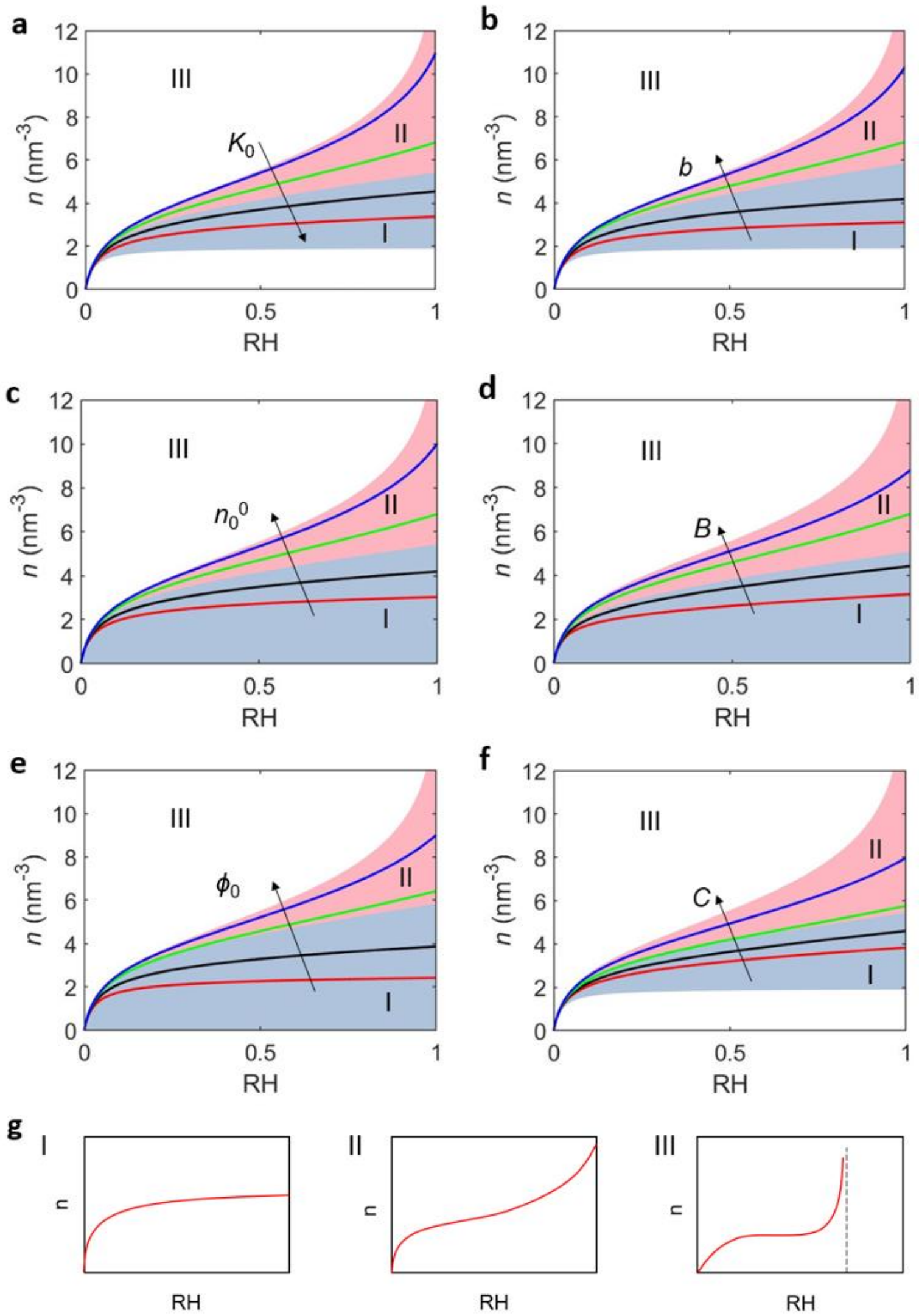
FIG. 8 Sorption isotherms at (a) different $K_{0}$ of $10 \mathrm{GPa}, 11 \mathrm{GPa}, 14 \mathrm{GPa}$ and $20 \mathrm{GPa}$; (b) different $b$ of 2.8, 7.4, 10.2 and 10.8; (c) different $n_{0}{ }^{0}$ of $0.8 \mathrm{~nm}^{-3}, 1.28 \mathrm{~nm}^{-3}, 1.76 \mathrm{~nm}^{-3}$ and $1.92 \mathrm{~nm}^{-3}$; (d) different $B$ of $0.0043 \mathrm{~Pa}^{-1}, 0.0130 \mathrm{~Pa}^{-1}, 0.0303 \mathrm{~Pa}^{-1}$ and $0.0389 \mathrm{~Pa}^{-1}$; (e) different $\phi_{0}$ of $0.03,0.06$, 0.075 and 0.079 ; (f) different $C$ of 9, 10, 11 and 12. (g) Schematics of three different types of sorption isotherm. The black arrow indicates the direction of increasing corresponding parameters. Regions I (gray), II (pink) and III (white) correspond to sorption isotherms indicated by I, II and III in $(\mathrm{g})$ respectively.

The other parameter influencing the mechanical property is $b$ which can be seen as the weakening parameter. With a larger $b$, the tangential stiffness decreases more as strain increases. The impact of $b$ on sorption isotherm is plotted in FIG. 8 (b). It shows that increasing $b$ has a similar effect as decreasing $K_{0}$, i.e., the sorption isotherm turns from Type I to Type II and the sorption amount increases. Indeed, larger $b$ and smaller $K_{0}$ have similar effects as both make the solid softer.

The effects of sorption related parameters, $n_{0}{ }^{0}$ and $B$, on sorption isotherm are plotted in FIG. 8 (c) and (d). $n_{0}{ }^{0}$ specifies how much water is adsorbed at saturated state, i.e. the sorption capacity for the undeformed solid, while $B$ specifies how 'fast' sorption proceeds at low pressure/RH. Thus, a system with higher $n_{0}{ }^{0}$ and $B$ corresponds to a stronger adsorbent-adsorbate interaction. FIG. 8 shows that, as $n_{0}{ }^{0}$ and $B$ increases, sorption amount increases at same RH. Larger $n_{0}{ }^{0}$ and $B$ lead to larger sorption-induced stress according to Eq. (13). As a result, the solid undergoes larger deformation and more additional pore space is generated, which finally leads to larger sorption amount. It also shows that the sorption isotherm changes from Type I to Type II as $n_{0}{ }^{0}$ and $B$ increases.

There are two parameters in the poromechanical model characterizing the structure of the system, $\phi_{0}$ and $C$. $\phi_{0}$ characterizes the initial porosity and $C$ characterizes the dependence of current porosity on the strain. In the previous part, $C$ was taken as $1 / \phi_{0}$ assuming that the solid does not deform and all the strain comes from the change in porosity. However, this does not essentially hold to be true for other nanoporous polymers. Here we give up this constraint and discuss the contributions of the two parameters independently. Sorption isotherms at different $\phi_{0}$ values are plotted in FIG. 8 (e). The ratio $n_{0} \% \phi_{0}$ is kept constant so that the sorption amount per unit pore volume is constant. It shows that increasing $\phi_{0}$ leads 
to an increase of the sorption and the change of sorption isotherm from Type I to Type II. This is because a system with larger initial porosity $\phi_{0}$ has larger sorption capacity $n_{0}{ }^{0}$ and thus has larger sorption amount, which leads to larger sorption-induced stress according to Eq. (13). Similar mechanism applies to the influence of $C$, which is plotted in FIG. 8 (f). As sorption-induced stress is proportional to $C$ according to Eq. (13), a larger $C$ also leads to larger sorption amount and change of sorption isotherm from type I to type II as seen when studying $\phi_{0}$.

As we see from the discussion above, material properties significantly impact the coupling behavior. However, each material property is characterized by more than one parameter. For instance, the stiffness of the material is characterized by both $K_{0}$ and $b$. Though discussion based on each parameter sheds light on the specific effect, it is inconvenient to have a whole picture of the influence of different material properties and draw general conclusions based on these discussions. Here we consider the combined effect of all the parameters characterizing the same class of material properties. For instance, both $K_{0}$ and $b$ characterize the mechanical properties of the solid, thus we consider the combined effect of $K_{0}$ and $b$. Instead of explicitly looking at the sorption isotherm, we concentrate more on the influence of the two parameters over the entire $\mathrm{RH}$ region from 0 to 1 . A good choice for an indicator is the free energy associated with sorption, i.e., the area under the curve when plotting sorption amount against chemical potential:

$$
f=-\int_{-\infty}^{\mu_{0}} n \mathrm{~d} \mu
$$

Combining Eqs. (9), (11) and (17) gives

$$
f=-R T n_{0}^{0} \ln \left(1+B p_{0}\right)-\frac{K_{0}}{b^{2}} e^{-b \phi_{0}}(Z \ln Z-Z+1)
$$


where $\mathrm{Z}=1-\frac{b}{K_{0}} C R \operatorname{Tn}_{0}^{0}\left(1+B p_{0}\right)$. A larger $f$ means a larger free energy associated with sorption and corresponds to the larger total sorption amount. Moreover, it also corresponds to a larger deformation magnitude because of the coupling between sorption and deformation. For concision, $f$ is normalized by $f_{0}$ in the following discussion, where $f_{0}$ is defined as the free energy corresponding to the case of zero strain. According to Eq. (9), the sorption amount with some swelling strain will always be larger than that of zero strain. Thus, $f / f_{0} \geq 1$ holds for all the swelling cases.
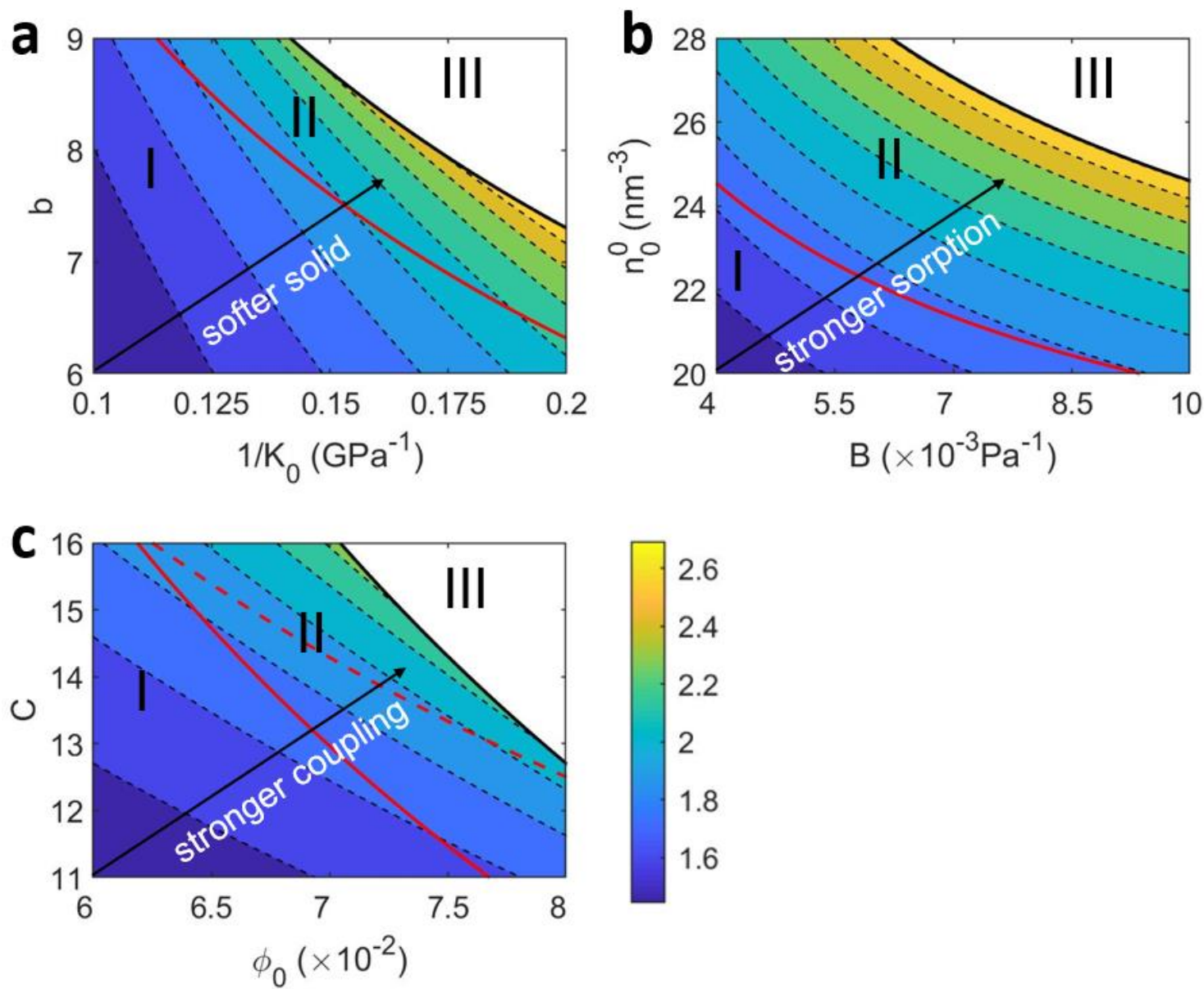

FIG. 9 Combined effects of (a) $K_{0}$ and $b$, (b) $n_{0}{ }^{0}$ and $B$ and (c) $\phi_{0}$ and $C$ on the sorption-related free energy $f$. The regions I, II and III divided by red and black solid lines correspond to the three scenarios given in FIG. 8(g). The red dashed line in (c) represents $C=1 / \phi_{0}$.

The influence of the stiffness is characterized by studying the combined effects of $K_{0}$ and $b$ as plotted in FIG. 9 (a). Note that the compliance, i.e., $1 / K_{0}$, is taken so that the stiffest 
solid sits on the origin. By going along the diagonal from origin to top right, the solid becomes softer and we see an increase in $f$. Indeed, the magnitude of deformation is larger for softer solid, which leads to a larger sorption amount. As a result, the free energy related to sorption is larger. We can also define three regions, I, II and III in a similar way as done in FIG. 8. Region I (down left of the red solid line) corresponds to stiff solids, the deformation of which is limited and thus corresponds to Type I isotherm. Region II (between the black and red solid lines) corresponds to softer solids and larger deformation, which gives Type II isotherm. Region III corresponds to the case where the solid is too soft to sustain the sorption-induced stress, thus RH could not reach 1.

Following the same line, the combined effect of $n_{0}{ }^{0}$ and $B$ is plotted in FIG. 9 (b) to characterize the influence of the adsorbent-adsorbate interaction strength. The strength increases by going along the diagonal from origin to top right, upon which we see more sorption amount as indicated by larger $f$ and sorption isotherm turns from type I to type II. Similarly, the combined effects of $\phi_{0}$ and $C$ are plotted in FIG. 9 (c). Though $\phi_{0}$ and $C$ are parameters related to the structure (porosity), they can also be viewed as parameters characterizing the coupling strength as the two parameters define the mutual influence of deformation and sorption in the poromechanical model. $\phi_{0}$ characterizes the influence of the sorption on deformation. A larger $\phi_{0}$ corresponds to a larger domain of fluid within the system, thus leads to a stronger influence of sorption on deformation. $C$ characterizes the influence of deformation on sorption. A larger $C$ corresponds to a larger additional pore space created by the strain thus leads to a larger sorption amount. Thus, for a system with larger $\phi_{0}$ and $C$, stronger coupling between sorption and deformation can be expected. Along this line, we can say that coupling turns from weak to strong by going along the diagonal of FIG. 9 (c) from origin to top right. The free energy $f$ gets larger for strong coupling. This is because deformation facilitates sorption as the swelling leads to a higher sorption amount. A stronger coupling leads to more sorption-related free energy. The line of $C=1 / \phi_{0}$ is also plotted as the red dashed line, which is the simplification we employ when addressing AC.

\subsection{Absence of Hysteresis}


We note that it is well-known that sorption in microporous polymers is accompanied with significant hysteresis. In this paper, however, only one sorption branch (desorption) is considered and hysteresis is not included into the poromechanical model. According to our research based on molecular simulations, the hysteresis is related to microscopic picture of hydrogen bonds (Chen et al., 2018). From the perspective of porous media, hysteresis relies on the mutual influence of the neighboring pores. Thus, we believe that a microscopic structure is needed for a physically-based model to account for hysteresis. However, poromechanics is a macroscopic approach, where the structural information of the whole system is represented by a scalar porosity and the microscopic structure is inherently absent. As a result, the poromechanical model proposed in this paper is incapable of modeling the hysteresis physically. We are working on this issue with an attempt to take the microscopic structure into account with the help of Dependent Domain model (Guyer et al., 2011).

\section{Conclusion}

In this work, the coupling of sorption and deformation in a soft nanoporous polymer was first investigated by means of molecular simulations. The atom-scale simulations, which are found to agree with experiments, reveal the coupling mechanism at the molecular level. We find that the Type II sorption isotherm of amorphous cellulose upon free swelling is induced by the sorption-induced deformation, i.e. the mechanosorptive effect. The sorption isotherm changes to Type I if no deformation is allowed by applying fixed displacement boundary conditions. Moreover, we find by means of molecular simulations that the stress state of the system has a significant impact on the sorption process. A tensile/compressive stress will facilitate/restrict the sorption.

Based on the molecular simulations reported in the present work, a poromechanical model was proposed. We demonstrate that the poromechanical model agrees with molecular simulations and experiments, and captures the main feature of the coupling mechanism. Three categories of parameters come into the poromechanical model: the mechanical 
properties, the sorption characteristics and the structural information. We find that different combinations of these parameters can lead to totally different sorption and deformation behaviors: the sorption isotherm can even change its type. Basically, systems with lower modulus, stronger adsorbent-adsorbate interaction and stronger coupling between sorption and deformation tend to have a Type II sorption isotherm with larger sorption amount and sorption-induced deformation, while systems with higher modulus, weaker adsorbentadsorbate interaction and weaker coupling between sorption and deformation tend to have a Type I sorption isotherm with limited sorption amount and sorption-induced deformation.

The poromechanical model shows its versatility in addressing the coupling between sorption and deformation of nanoporous polymers. Compared to molecular simulations, it can easily characterize the individual contributions of each physical property, which deepens our understanding of the coupled behavior. Besides, it is efficient and simple compared to the molecular simulations without loss of major coupling mechanism. Thus, this poromechanical model can be viewed as an upscaled model for nanoporous polymers at nanoscale, which can help to design and tailor materials for target coupled behaviors. Moreover, it can also be easily applied to studies of polymer-based materials with more complex microstructures (Safari and van de Ven, 2016).

\section{Acknowledgements}

The authors acknowledge the support of the Swiss National Science Foundation (SNSF) (No.143601).

\section{References}

Anand, L., 2017. A large deformation poroplasticity theory for microporous polymeric materials. J. Mech. Phys. Solids 98, 126-155. https://doi.org/10.1016/j.jmps.2016.07.017 
Bakhshian, S., Sahimi, M., 2018. Theoretical Model and Numerical Simulation of Adsorption and Deformation in Flexible Metal-Organic Frameworks. J. Phys. Chem. C 122, 9465-9473. https://doi.org/10.1021/acs.jpcc.8b00924

Balzer, C., Waag, A.M., Gehret, S., Reichenauer, G., Putz, F., Hüsing, N., Paris, O., Bernstein, N., Gor, G.Y., Neimark, A. V., 2017. Adsorption-Induced Deformation of Hierarchically Structured Mesoporous Silica-Effect of Pore-Level Anisotropy. Langmuir 33, 5592-5602. https://doi.org/10.1021/acs.langmuir.7b00468

Barreiro, J.A., Minichini, A., Barreiro, J.A., Sandoval, A.J., 2010. Water Sorption Isotherms of NPK 10-20-20/4 Muriate of Potash Fertilizer. Ind. Eng. Chem. Res. 49, 887-892. https://doi.org/10.1021/ie900533q

Bharadwaj, N.A., Schweizer, K.S., Ewoldt, R.H., 2017. A strain stiffening theory for transient polymer networks under asymptotically nonlinear oscillatory shear. J. Rheol. (N. Y. N. Y). 61, 643-665. https://doi.org/10.1122/1.4979368

Brochard, L., Vandamme, M., Pellenq, R.J.-M., 2012. Poromechanics of microporous media. J. Mech. Phys. Solids 60, 606-622. https://doi.org/10.1016/j.jmps.2012.01.001

Chen, M., Coasne, B., Guyer, R., Derome, D., Carmeliet, J., 2019. Molecular Simulation of Sorption-Induced Deformation in Atomistic Nanoporous Materials. Langmuir 35, 7751-7758. https://doi.org/10.1021/acs.langmuir.9b00859

Chen, M., Coasne, B., Guyer, R., Derome, D., Carmeliet, J., 2018. Role of hydrogen bonding in hysteresis observed in sorption-induced swelling of soft nanoporous polymers. Nat. Commun. 9, 3507. https://doi.org/10.1038/s41467-018-05897-9

Chen, W., Lickfield, G.C., Yang, C.Q., 2004. Molecular modeling of cellulose in 
amorphous state. Part I: model building and plastic deformation study. Polymer (Guildf). 45, 1063-1071. https://doi.org/10.1016/j.polymer.2003.11.020

Coasne, B., Alba-Simionesco, C., Audonnet, F., Dosseh, G., Gubbins, K.E., 2007. Molecular simulation of the adsorption and structure of benzene confined in mesoporous silicas. Adsorption 13, 485-490. https://doi.org/10.1007/s10450-0079051-3

Coasne, B., Weigel, C., Polian, A., Kint, M., Rouquette, J., Haines, J., Foret, M., Vacher, R., Rufflé, B., 2014. Poroelastic Theory Applied to the Adsorption-Induced Deformation of Vitreous Silica. J. Phys. Chem. B 118, 14519-14525. https://doi.org/10.1021/jp5094383

Dawson, C., Vincent, J.F. V., Rocca, A.-M., 1997. How pine cones open. Nature 390, 668-668. https://doi.org/10.1038/37745

Derome, D., Rafsanjani, A., Patera, A., Guyer, R., Carmeliet, J., 2012. Hygromorphic behaviour of cellular material: hysteretic swelling and shrinkage of wood probed by phase contrast X-ray tomography. Philos. Mag. 92, 3680-3698. https://doi.org/10.1080/14786435.2012.715248

Dollimore, D., Heal, G.R., 2007. An improved method for the calculation of pore size distribution from adsorption data. J. Appl. Chem. 14, 109-114. https://doi.org/10.1002/jctb.5010140302

Flory, P.J., Rehner, J., 1943. Statistical Mechanics of Cross- Linked Polymer Networks II. Swelling. J. Chem. Phys. 11, 521-526. https://doi.org/10.1063/1.1723792

Fomkin, A.A., 2005. Adsorption of Gases, Vapors and Liquids by Microporous Adsorbents. Adsorption 11, 425-436. https://doi.org/10.1007/s10450-005-5636-x 
Gor, G.Y., Bernstein, N., 2016. Revisiting Bangham's law of adsorption-induced deformation: changes of surface energy and surface stress. Phys. Chem. Chem. Phys. 18, 9788-9798. https://doi.org/10.1039/C6CP00051G

Gor, G.Y., Huber, P., Bernstein, N., 2017. Adsorption-induced deformation of nanoporous materials—A review. Appl. Phys. Rev. 4, 011303. https://doi.org/10.1063/1.4975001

Gor, G.Y., Huber, P., Weissmüller, J., 2018. Elastocapillarity in nanopores: Sorption strain from the actions of surface tension and surface stress. Phys. Rev. Mater. 2, 086002. https://doi.org/10.1103/PhysRevMaterials.2.086002

Gor, G.Y., Neimark, A. V, 2011. Adsorption-Induced Deformation of Mesoporous Solids: Macroscopic Approach and Density Functional Theory. Langmuir 27, 69266931. https://doi.org/10.1021/la201271p

Gor, G.Y., Paris, O., Prass, J., Russo, P. a., Ribeiro Carrott, M.M.L., Neimark, A. V., 2013. Adsorption of n-Pentane on Mesoporous Silica and Adsorbent Deformation. Langmuir 29, 8601-8608. https://doi.org/10.1021/la401513n

Groen, J.C., Peffer, L.A.., Pérez-Ramírez, J., 2003. Pore size determination in modified micro- and mesoporous materials. Pitfalls and limitations in gas adsorption data analysis. Microporous Mesoporous Mater. 60, 1-17. https://doi.org/10.1016/S13871811(03)00339-1

Günther, G., Prass, J., Paris, O., Schoen, M., 2008. Novel Insights into Nanopore Deformation Caused by Capillary Condensation. Phys. Rev. Lett. 101, 086104. https://doi.org/10.1103/PhysRevLett.101.086104

Guyer, R. a., Kim, H.A., Derome, D., Carmeliet, J., TenCate, J., 2011. Hysteresis in 
modeling of poroelastic systems: Quasistatic equilibrium. Phys. Rev. E 83, 061408. https://doi.org/10.1103/PhysRevE.83.061408

Hahn, H.T., 1976. Residual Stresses in Polymer Matrix Composite Laminates. J. Compos. Mater. 10, 266-278. https://doi.org/10.1177/002199837601000401

Jaspers, M., Dennison, M., Mabesoone, M.F.J., MacKintosh, F.C., Rowan, A.E., Kouwer, P.H.J., 2014. Ultra-responsive soft matter from strain-stiffening hydrogels. Nat. Commun. 5, 5808. https://doi.org/10.1038/ncomms6808

Jin, D., Lu, X., Zhang, M., Wei, S., Zhu, Q., Shi, X., Shao, Y., Wang, W., Guo, W., 2014. The adsorption behaviour of $\mathrm{CH} 4$ on microporous carbons: effects of surface heterogeneity. Phys. Chem. Chem. Phys. 16, 11037. https://doi.org/10.1039/c3cp55107e

Kulasinski, K., Guyer, R., Derome, D., Carmeliet, J., 2015a. Poroelastic model for adsorption-induced deformation of biopolymers obtained from molecular simulations. Phys. Rev. E 92, 022605. https://doi.org/10.1103/PhysRevE.92.022605

Kulasinski, K., Guyer, R., Keten, S., Derome, D., Carmeliet, J., 2015b. Impact of Moisture Adsorption on Structure and Physical Properties of Amorphous Biopolymers. Macromolecules 48, 2793-2800. https://doi.org/10.1021/acs.macromol.5b00248

Kulasinski, K., Keten, S., Churakov, S. V., Derome, D., Carmeliet, J., 2014a. A comparative molecular dynamics study of crystalline, paracrystalline and amorphous states of cellulose. Cellulose 21, 1103-1116. https://doi.org/10.1007/s10570-0140213-7

Kulasinski, K., Keten, S., Churakov, S. V., Guyer, R., Carmeliet, J., Derome, D., 2014 b. 
Molecular Mechanism of Moisture-Induced Transition in Amorphous Cellulose. ACS Macro Lett. 3, 1037-1040. https://doi.org/10.1021/mz500528m

Lai, W.M., Hou, J.S., Mow, V.C., 1991. A Triphasic Theory for the Swelling and Deformation Behaviors of Articular Cartilage. J. Biomech. Eng. 113, 245-258. https://doi.org/10.1115/1.2894880

Lee, Y., Kim, S.-K., Park, Y.-J., Cho, J., Koo, H.-J., 2019. A Humidity-Sensing Composite Microfiber Based on Moisture-Induced Swelling of an Agarose Polymer Matrix. Polym. Compos. https://doi.org/10.1002/pc.25220

Loh, W.K., Crocombe, A.D., Abdel Wahab, M.M., Ashcroft, I.A., 2005. Modelling anomalous moisture uptake, swelling and thermal characteristics of a rubber toughened epoxy adhesive. Int. J. Adhes. Adhes. 25, 1-12. https://doi.org/10.1016/j.jjadhadh.2004.02.002

Long, Y., Palmer, J.C., Coasne, B., Śliwinska-Bartkowiak, M., Gubbins, K.E., 2012. Under pressure: Quasi-high pressure effects in nanopores. Microporous Mesoporous Mater. 154, 19-23. https://doi.org/10.1016/j.micromeso.2011.07.017

Meng, Q.B., Weber, J., 2014. Lignin-based Microporous Materials as Selective Adsorbents for Carbon Dioxide Separation. ChemSusChem 7, 3312-3318. https://doi.org/10.1002/cssc.201402879

Mihranyan, A., Llagostera, A.P., Karmhag, R., Strømme, M., Ek, R., 2004. Moisture sorption by cellulose powders of varying crystallinity. Int. J. Pharm. 269, 433-442. https://doi.org/10.1016/j.ijpharm.2003.09.030

Neimark, A. V., Coudert, F.-X., Boutin, A., Fuchs, A.H., 2010. Stress-Based Model for the Breathing of Metal-Organic Frameworks. J. Phys. Chem. Lett. 1, 445-449. 
https://doi.org/10.1021/jz9003087

Park, H.B., Jung, C.H., Lee, Y.M., Hill, A.J., Pas, S.J., Mudie, S.T., Van Wagner, E., Freeman, B.D., Cookson, D.J., 2007. Polymers with Cavities Tuned for Fast Selective Transport of Small Molecules and Ions. Science (80-. ). 318, 254-258. https://doi.org/10.1126/science.1146744

Plimpton, S., 1995. Fast Parallel Algorithms for Short-Range Molecular Dynamics. J. Comput. Phys. 117, 1-19. https://doi.org/10.1006/jcph.1995.1039

Ryckaert, J.-P., Ciccotti, G., Berendsen, H.J.C., 1977. Numerical integration of the cartesian equations of motion of a system with constraints: molecular dynamics of $\mathrm{n}$ alkanes. J. Comput. Phys. 23, 327-341. https://doi.org/10.1016/00219991(77)90098-5

Safari, S., van de Ven, T.G.M., 2016. Effect of Water Vapor Adsorption on Electrical Properties of Carbon Nanotube/Nanocrystalline Cellulose Composites. ACS Appl. Mater. Interfaces 8, 9483-9489. https://doi.org/10.1021/acsami.6b02374

Schlaich, A., Coasne, B., 2019. Dispersion truncation affects the phase behavior of bulk and confined fluids: Coexistence, adsorption, and criticality. J. Chem. Phys. 150, 154104. https://doi.org/10.1063/1.5085431

Sing, K.S.W., 1985. Reporting physisorption data for gas/solid systems with special reference to the determination of surface area and porosity (Recommendations 1984). Pure Appl. Chem. 57, 603-619. https://doi.org/10.1351/pac198557040603

Sun, H., Mumby, S.J., Maple, J.R., Hagler, A.T., 1994. An ab Initio CFF93 All-Atom Force Field for Polycarbonates. J. Am. Chem. Soc. 116, 2978-2987. https://doi.org/10.1021/ja00086a030 
Thommes, M., Kaneko, K., Neimark, A. V., Olivier, J.P., Rodriguez-Reinoso, F., Rouquerol, J., Sing, K.S.W., 2015. Physisorption of gases, with special reference to the evaluation of surface area and pore size distribution (IUPAC Technical Report). Pure Appl. Chem. 87, 1051-1069. https://doi.org/10.1515/pac-2014-1117

Vandamme, M., 2019. Coupling between adsorption and mechanics (and vice versa). Curr. Opin. Chem. Eng. 24, 12-18. https://doi.org/10.1016/j.coche.2018.12.005

Vermorel, R., Pijaudier-Cabot, G., 2014. Enhanced continuum poromechanics to account for adsorption induced swelling of saturated isotropic microporous materials. Eur. J. Mech. - A/Solids 44, 148-156. https://doi.org/10.1016/j.euromechsol.2013.10.010

Weber, J., Antonietti, M., Thomas, A., 2008. Microporous Networks of HighPerformance Polymers: Elastic Deformations and Gas Sorption Properties. Macromolecules 41, 2880-2885. https://doi.org/10.1021/ma702495r

Xiang, Y., Zhong, D., Wang, P., Yin, T., Zhou, H., Yu, H., Baliga, C., Qu, S., Yang, W., 2019. A physically based visco-hyperelastic constitutive model for soft materials. J. Mech. Phys. Solids 128, 208-218. https://doi.org/10.1016/j.jmps.2019.04.010

Yakovlev, V.Y., Fomkin, A.A., Tvardovski, A.V., 2004. Adsorption and deformation phenomena at interaction of $\mathrm{N} 2$ and microporous carbon adsorbent. J. Colloid Interface Sci. 280, 305-308. https://doi.org/10.1016/j.jcis.2004.07.029

Yang, X., Wang, W., Miao, M., 2018. Moisture-Responsive Natural Fiber CoilStructured Artificial Muscles. ACS Appl. Mater. Interfaces 10, 32256-32264. https://doi.org/10.1021/acsami.8b12144

Yu, C., Malakpoor, K., Huyghe, J.M., 2018. A three-dimensional transient mixed hybrid finite element model for superabsorbent polymers with strain-dependent 
permeability. Soft Matter 14, 3834-3848. https://doi.org/10.1039/C7SM01587A

Zhang, Y., 2018. Mechanics of adsorption-deformation coupling in porous media. J. Mech. Phys. Solids 114, 31-54. https://doi.org/10.1016/j.jmps.2018.02.009

Zhao, F., Zhou, X., Liu, Y., Shi, Y., Dai, Y., Yu, G., 2019. Super Moisture-Absorbent Gels for All-Weather Atmospheric Water Harvesting. Adv. Mater. 31, 1806446. https://doi.org/10.1002/adma.201806446 